\title{
Self-Other Representation in the Social Brain Reflects Social Connection
}

\author{
${ }^{(10}$ Andrea L. Courtney ${ }^{1}$ and Meghan L. Meyer ${ }^{2}$ \\ ${ }^{1}$ Department of Psychology, Stanford University, Stanford, California 94305, and ${ }^{2}$ Department of Psychological \& Brain Sciences, Dartmouth \\ College, Hanover, New Hampshire 03755
}

Social connection is critical to well-being, yet how the brain reflects our attachment to other people remains largely unknown. We combined univariate and multivariate brain imaging analyses to assess whether and how the brain organizes representations of others based on how connected they are to our own identity. During an fMRI scan, female and male human participants $(N=43)$ completed a self- and other-reflection task for 16 targets: the self, five close others, five acquaintances, and five celebrities. In addition, they reported their subjective closeness to each target and their own trait loneliness. We examined neural responses to the self and others in a brain region that has been associated with self-representation (mPFC) and across the whole brain. The structure of self-other representation in the mPFC and across the social brain appeared to cluster targets into three social categories: the self, social network members (including close others and acquaintances), and celebrities. Moreover, both univariate activation in mPFC and multivariate self-other similarity in mPFC and across the social brain increased with subjective self-other closeness ratings. Critically, participants who were less socially connected (i.e., lonelier) showed altered self-other mapping in social brain regions. Most notably, in mPFC, loneliness was associated with reduced representational similarity between the self and others. The social brain apparently maintains information about broad social categories as well as closeness to the self. Moreover, these results point to the possibility that feelings of chronic social disconnection may be mirrored by a "lonelier" neural self-representation.

Key words: mPFC; self; social; brain; social cognition; social neuroscience

Significance Statement

Social connection is critical to well-being, yet how the brain reflects our attachment to people remains unclear. We found that the social brain stratifies neural representations of people based on our subjective connection to them, separately clustering people who are and are not in our social network. Moreover, the people we feel closest to are represented most closely to ourselves. Finally, lonelier individuals also appeared to have a "lonelier" neural self-representation in the mPFC, as loneliness attenuated the similarity between self and other neural representations in this region. The social brain appears to map our interpersonal ties, and alterations in this map may help explain why lonely individuals endorse statements such as "people are around me but not with me."

\section{Introduction}

Feeling close to other people promotes well-being (Barnett and Gotlib, 1988; Diener and Seligman, 2002; Holt-Lunstad et al., 2010), whereas feeling disconnected from them can compromise mental and physical health (Baumeister and Tice, 1990;

\footnotetext{
Received Nov. 27, 2019; revised Mar. 22, 2020; accepted Apr. 29, 2020.

Author contributions: A.L.C. and M.L.M. designed research; A.L.C. performed research; A.L.C. analyzed data; A.L.C. and M.L.M. wrote the paper.

The authors declare no competing financial interests.

This work was supported by National Institutes of Health, National Institute of Mental Health Award F31MH111192. The content is solely the responsibility of the authors and does not necessarily represent the official views of the National Institutes of Health.

Correspondence should be addressed to Andrea L. Courtney at acourtne@stanford.edu.

https://doi.org/10.1523/JNEUROSCI.2826-19.2020

Copyright ( 2020 the authors
}

Baumeister and Leary, 1995; Cacioppo et al., 2006). Yet, how the brain represents interpersonal closeness remains unclear. Filling this gap is critical as it may reveal basic mechanisms to intervene on to increase subjective connection and in turn promote well-being.

Insight into how the brain represents subjective social connection may come from a close examination of the mPFC. While the $\mathrm{mPFC}$ is known to preferentially activate in response to thinking about the self, it exhibits similar activation when thinking about close others (Seger et al., 2004; Heatherton et al., 2006; Krienen et al., 2010; Moran et al., 2011; Chen et al., 2013). Moreover, these activation levels persist after controlling for one's similarity to the close other considered (Krienen et al., 2010), and are elicited more strongly by deeper characteristics of the person (e.g., their personality) than by superficial 
characteristics (e.g., their appearance) (Moran et al., 2011). Collectively, these results suggest that the mPFC may play a key role in representing our personal connection to others.

If the mPFC represents our social connection to others, there are at least two ways in which it may pull this off. One possibility is that the mPFC keeps a structured map of our social circles, with people organized by how close they are to us (Dunbar, 2018). Consistent with this possibility, mPFC responses reflect others' objective social network positions (Parkinson et al., 2017). For example, multivariate mPFC responses to viewing people from one's own social network mirror their eigenvector centrality (i.e., the extent to which they are connected to wellconnected others in a social network, a metric of objective popularity). By extension, the mPFC may also cluster representation of others based on how subjectively close we feel to them (i.e., as "cliques" varied by closeness). Another possibility is that interpersonal closeness impinges on our own self-representations, with closer individuals more similarly represented to ourselves. Indeed, social psychology suggests that self-representations include representations of close others (i.e., "self-other overlap") to foster social connection (Aron et al., 1991). Consistent with this possibility, friendship has been associated with sharing similar neural responses to the same social stimuli (Kang et al., 2010; Parkinson et al., 2018), suggesting that interpersonal closeness is tied to self-other similarity. Our first goal was to assess these two possibilities to determine whether and how the mPFC may represent the subjective connection between the self and others.

If the $\mathrm{mPFC}$ organizes self and other representations in either of these ways, a corollary question is whether a chronic lack of subjective social connection, as in the case of loneliness, may be linked to alterations in this structure. Loneliness is characterized by a perceived gap between the self and others: although many lonely individuals maintain multiple social relationships, they still experience loneliness because of the perception that they are disconnected from others (Jones, 1981; Berscheid and Reis, 1998; Binder et al., 2012; Russell et al., 2012). Thus, a second goal of this study was to examine whether loneliness is associated with altered self-other representation in the $\mathrm{MPFC}$, which might provide insight into this discrepancy. If the mPFC keeps an organized map of our social circles, loneliness may be associated with alterations in this map. Most notably, loneliness may be reflected by a lonelier "neural self," with less similarity in representations between the self and others.

To test these possibilities, we assessed neural responses during a self- and other-reflection task, and richly sampled social targets that varied in subjective closeness to participants. We were therefore able to test whether the mPFC (1) keeps an organized map of our interpersonal connections and/or (2) represents the self and others more similarly as a function of subjective closeness, and whether (3) loneliness modulates these patterns.

\section{Materials and Methods}

\section{Participants}

Fifty college students and community members (30 female) between the ages of 18 and 47 years (mean \pm SD, $20.2 \pm 4.6$ years) participated in this study. All participants were screened for compliance with MRI safety, reported normal neurologic history, and had normal or corrected-to-normal visual acuity. Each participant provided informed consent in accordance with the guidelines set by the Committee for the Protection of Human Subjects at Dartmouth College and received monetary compensation or class credit for participating in the study. fMRI data were excluded for participants $(n=7)$ whose movement during any run of the scan exceeded $3 \mathrm{~mm}$ in translation or 2 degrees in rotation.

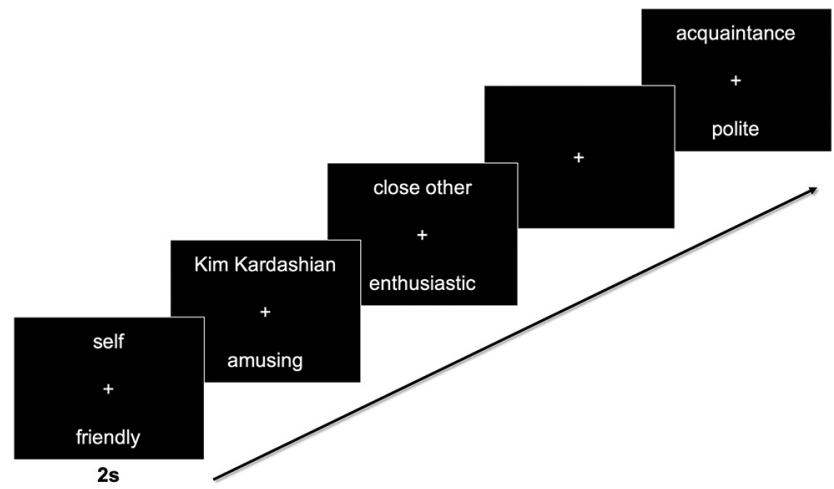

Figure 1. Schematic of self- and other-reflection task design. Participants considered the personality traits of the self, 5 close others, 5 acquaintances, and 5 celebrities across 10 runs. Each trial was presented for $2 \mathrm{~s}$ and was jittered with fixation.

Two additional participants did not complete the Revised UCLA Loneliness Scale (Russell et al., 1980) and were excluded from analyses requiring that measure.

\section{Experimental design and statistical analysis}

Procedure. Before the scan, participants completed a short survey in which they provided the names of (1) five close others with whom they had "the closest, deepest, most involved, and most intimate relationships" and (2) five acquaintances (e.g., classmates, colleagues, or neighbors), ranked in the order in which they felt closest to them. These names were used in the fMRI self- and other-reflection (i.e., trait judgment) task to elicit activation associated with thinking about close others and acquaintances.

During the scan, participants made trait judgments for 16 targets: the self, 5 nominated close others, 5 nominated acquaintances, and 5 wellknown celebrities (Ellen Degeneres, Kim Kardashian, Barack Obama, Justin Bieber, and Mark Zuckerberg), which have been used in similar studies (e.g., Thornton and Mitchell, 2017). Importantly, all targets were familiar to the participants but were expected to vary in subjective closeness. During each $2 \mathrm{~s}$ trial, one target name was presented above and a trait adjective presented below a central fixation cross. Participants were instructed to consider and respond with how much the trait describes the person ( $1=$ "not at all," 4 = "very much") using a button-box. The task lasted for 10 functional runs ( 80 trials each, 5 trials per target) for a total of 800 trials (Fig. 1).

Following the scan, participants rated their subjective closeness, similarity, and familiarity to each of the targets on a 1-100 scale $(1=$ "not at all," 100 = "very much"). Consistent with the idea that people feel closer to their close others, versus acquaintances, versus known celebrities, there was a linear trend in ratings of closeness toward each target, such that close others (mean $\pm \mathrm{SD}, 82.7 \pm 15.3$ ) were rated closer than acquaintances $(46.3 \pm 22.2)$, who were rated closer than celebrities $\left(7.6 \pm 12.6, B=-53.11, t_{(698)}=56.81, p<0.001\right)$. Likewise, close others were rated as more similar $(69.1 \pm 20.4)$ and familiar $(83.8 \pm 17.2)$ than acquaintances, who were as rated more similar $(43.7 \pm 23.6)$ and famil$\operatorname{iar}(48.6 \pm 24.2)$ than celebrities $\left(\mathrm{M}_{\text {similarity }}=14.2, \mathrm{SD}_{\text {similarity }}=16.9\right.$, $\left.\mathrm{M}_{\text {familiarity }}=33.56, \mathrm{SD}_{\text {familiarity }}=26.6\right)$. Because these ratings were all highly correlated (all $r$ values $>0.71$ ), it was not possible to assess their unique contributions to neural responses. Consequently, given past research suggesting that $\mathrm{mPFC}$ is more sensitive to closeness than these other, related variables (Krienen et al., 2010), paired with our specific predictions about closeness, only closeness ratings were used in the analyses presented here. In addition to these ratings, participants also completed the Revised UCLA Loneliness Scale $(41.8 \pm 9.4)$ (Russell et al., 1980).

Apparatus. Imaging data were acquired on a 3T Siemens MAGNETOM Prisma Scanner (Siemens) with a 32-channel head coil. Stimuli were presented from a 13-inch Apple MacBook Air laptop computer running PsychoPy version 1.85 software (Peirce, 2008). An Epson (model ELP-7000) LCD projector displayed the stimuli on a screen at 
A

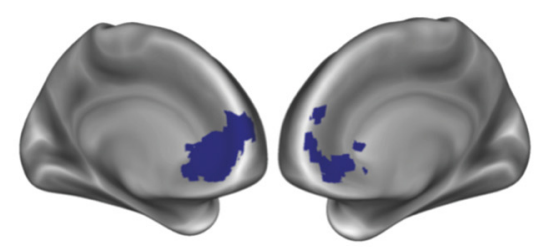

B

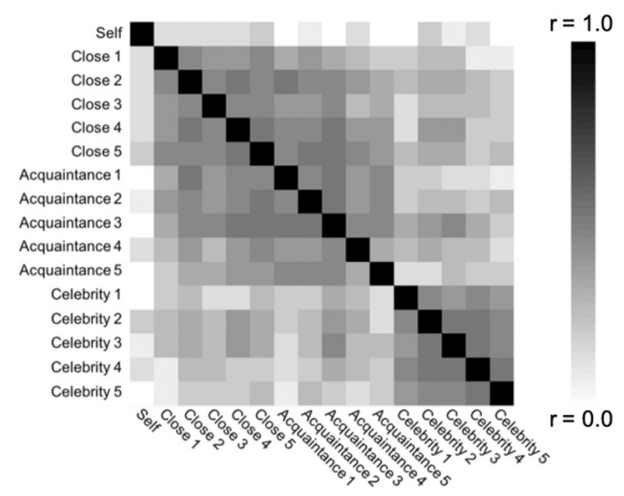

C

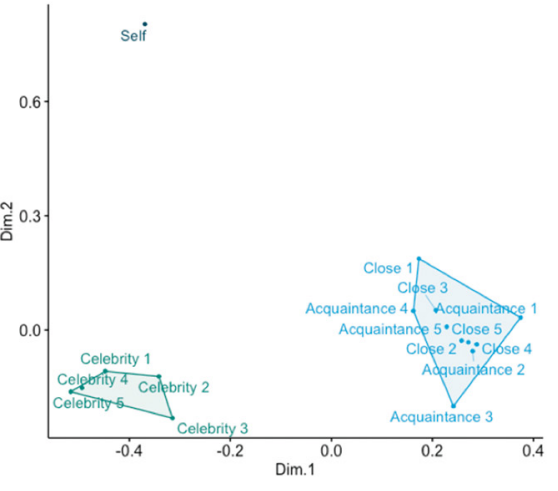

Figure 2. A, ROI mask of the mPFC created from a meta-analytic search for the term "self," restricted to BA 10 coordinates (Yarkoni et al., 2011). B, RSA of the self and others in the mPFC ROI reflected in a correlation matrix. C, A multidimensional scaling plot depicting correlation distances in the mPFC on two explanatory dimensions.

the head end of the scanner bore. Subjects viewed that screen through a mirror mounted on top of the head coil.

fMRI image acquisition. An anatomic (T1) image was acquired using a high-resolution 3-D MPRAGE sequence $(\mathrm{TR}=9.9 \mathrm{~ms}$; $\mathrm{TE}=4.6 \mathrm{~ms}$; flip angle $=8^{\circ} ; 1 \times 1 \times 1 \mathrm{~mm}^{3}$ voxels). Functional images were collected using a $\mathrm{T} 22^{*}$-weighted EPI sequence $(\mathrm{TR}=1000 \mathrm{~ms}$, TE $=30 \mathrm{~ms}$, flip angle $=59^{\circ}$, bandwidth $=2742$, echo spacing $=0.49,2.5 \times 2.5 \times 2.5 \mathrm{~mm}$ resolution) with a simultaneous multi-slice of four and generalized autocalibrating partial parallel acquisition of 1 . Ten functional runs of 250 axial images (52 slices, $130 \mathrm{~mm}$ coverage) were acquired for each participant. Sequence optimization was obtained using optseq2 (Dale, 1999) and included $30 \%$ jittered trials of fixation for obtaining a baseline estimation of neural activity.

$f M R I$ preprocessing and response estimation. Univariate functional neuroimaging data were analyzed using SPM12 (Wellcome Department of Cognitive Neurology, London) in conjunction with a suite of preprocessing and analysis tools (https://github.com/wagner-lab/spm12w). Functional data were slice time-corrected, realigned within and across runs to correct for head movement, and transformed into a standard anatomic space ( $3 \mathrm{~mm}$ isotropic voxels) based on the ICBM 152 brain template space (MNI). Normalized data were then smoothed spatially using an $8 \mathrm{~mm}$ FWHM Gaussian kernel for univariate analyses and a 4 mm FWHM Gaussian kernel for multivariate analyses. To further account for motion artifact, participants that demonstrated substantial movement $(>3 \mathrm{~mm}$ in translation or 2 degrees in rotation) were discarded ( $\mathrm{n}=7$, as noted above in Participants).

A GLM incorporating task effects (modeled as events of interest convolved with the canonical hemodynamic response function) was used to compute $\beta$ images estimating task-related effects for every voxel in the brain. The model included nuisance regressors for six motion parameters $(x, y, z$ directions and roll, pitch, yaw rotations), a linear drift, and run constants. The resulting $\beta$ images were used to compute a wholebrain voxelwise contrast comparing each target with the baseline condition (fixation crosshair trials).

Multivariate analyses were conducted using Python tools for neuroimaging, including the PyMVPA toolkit (http://www.pymvpa.org) (Hanke et al., 2009) and SciPy (http://www.scipy.org). Voxelwise GLM $\beta$ images were used to conduct representational similarity analysis (RSA) (Kriegeskorte et al., 2008) for the comparison of activation patterns and information overlap across conditions. To interrogate the structure of self-other representation by investigating the (dis)similarity of activation profiles across conditions, a representational (dis)similarity matrix (RDM) was extracted from ROIs (for more details, see ROI specification). In addition, a sphere searchlight ( $3 \mathrm{~mm}$ radius) RSA was conducted to identify regions of the brain that best reflected (1) the (dis) similarity structure identified by the ROI analysis, which distinguished the self, social network members, and celebrities; and (2) self-other closeness ratings. For each searchlight, a one-sample $t$ test was conducted on the voxel-level Fisher $z$-transformed correlation values representing similarity of the neural and target RDMs. The resulting group-level statistical maps were voxelwise-thresholded at $p<0.001$ and cluster-corrected to $p<0.001$ (minimum extent threshold: $k=195$ contiguous voxels for the searchlight analysis of the ROI (dis)similarity structure; $k=157$ voxels for the self-other closeness searchlight analysis), as recommended by AFNI's 3dClustSim, using the spatial autocorrelation function (see Eklund et al., 2016; Cox et al., 2017).

ROI specification. Given our specific predictions about the role of the $\mathrm{mPFC}$ in reflecting connections between the self and others from our social circles, we defined an ROI to test the involvement and structure of self-other representation in this region. To define this ROI independently of our own data, we downloaded an association test $(z>3.0, p<$ 0.01) map generated by Neurosynth (Yarkoni et al., 2011), reflecting meta-analytic association with the term "self" (downloaded April 2018). Past work suggests that mPFC voxels in Brodmann area 10 in particular are associated with self-representation (Denny et al., 2012; Wagner et al., 2012; Meyer and Lieberman, 2018; Lieberman et al., 2019). Thus, we further restricted the mPFC ROI to coordinates in BA 10: in the $x$ dimension from -18 to 18 , in the $y$ dimension from 30 to 80 , and in the $z$ dimension from -12 to 22 , for a resulting mask size of 404 voxels (Fig. $2 A$ ). As shown in Results, our whole-brain analyses additionally implicated the posterior cingulate in neural self-other overlap. We therefore defined an a posteriori ROI for the posterior cingulate cortex (PCC) using the same approach described for the MPFC, to additionally probe how neural self-other overlap in this region relates to loneliness. That is, we used the same meta-analytic map for "self" and further restricted the mask in the $x$ dimension from -18 to 18 , in the $y$ dimension from -70 to -32 , and in the $z$ dimension from 6 to 44 to isolate the PCC, for a resulting mask size of 223 voxels.

Analysis of social category representation in $m P F C$. To examine whether mPFC keeps a structured map of the self and others from our social circles, a neural RDM was extracted from the mPFC ROI. This approach allowed us to compare the similarity of multivariate activation patterns associated with each target category (i.e., the self, close others, acquaintances, celebrities). We subsequently compared it with a descriptive, theoretical RDM that appeared to best match the mPFC pattern, distinguishing the self, social network members, and celebrities (see Results). That is, for each subject, the MFPC RDM was correlated with the theoretical RDM, and these individual Fisher $z$-transformed correlation values were submitted to a group-level $t$ test. To identify additional brain regions whose response patterns reflected the social category structure identified in the $\mathrm{mPFC}$ ROI, a sphere searchlight ( $3 \mathrm{~mm}$ radius) was used to conduct a whole-brain RSA with this structure as the model (Fig. $3 A)$.

Modulation of neural responses by self-other closeness. To determine whether neural responses in the MPFC were sensitive to subjective closeness between the self and others, we performed both univariate and multivariate analyses with the mPFC ROI. We extracted both univariate and multivariate $\beta$ estimates from the mPFC ROI and for both: (1) searched 
A

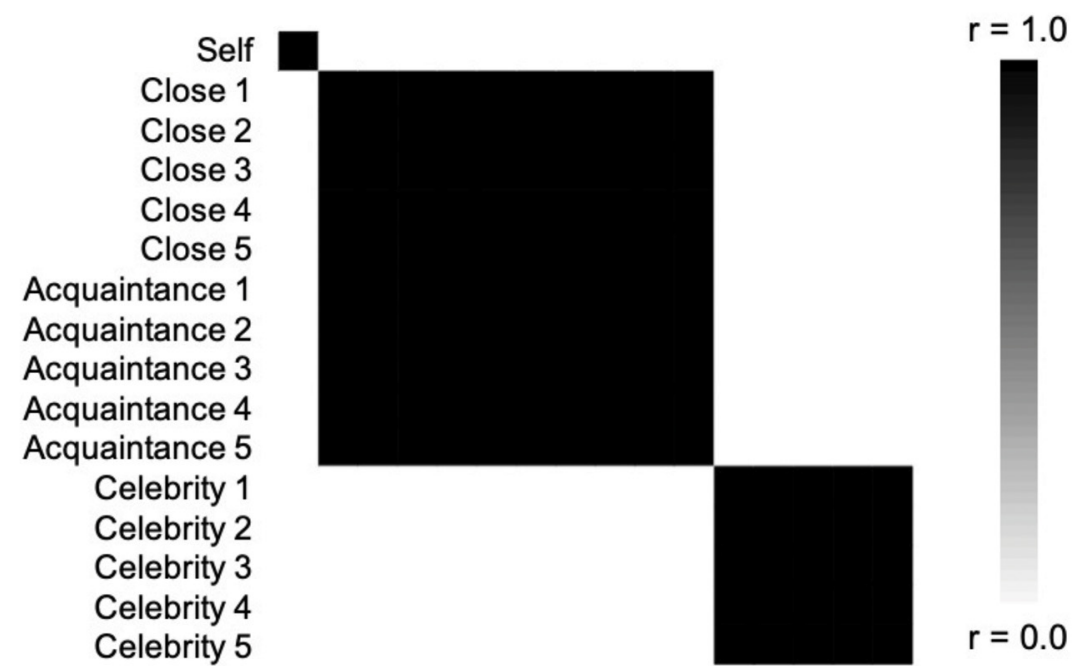

B
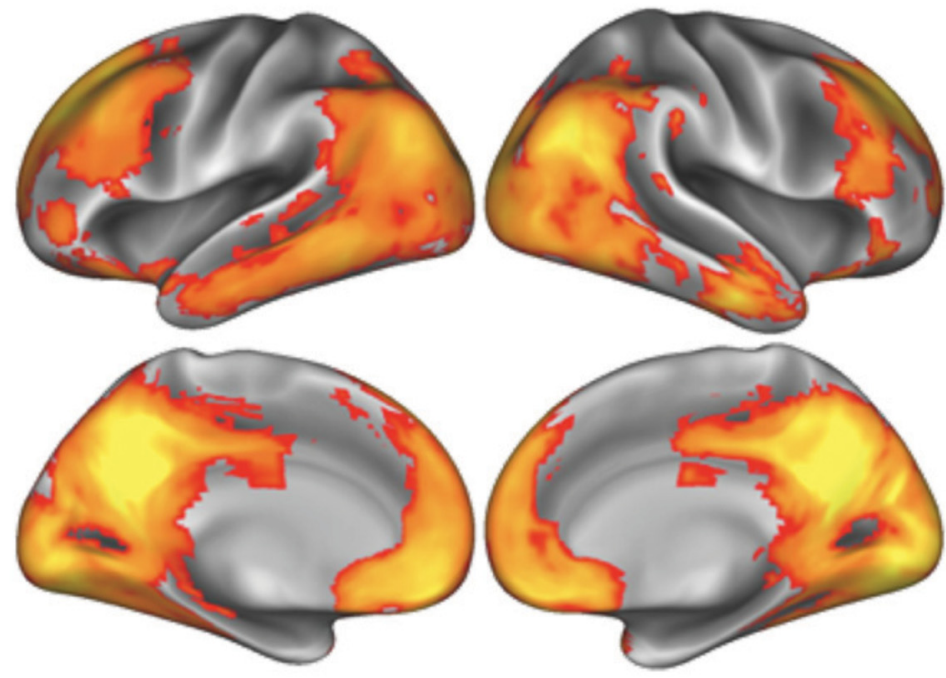

Figure 3. $\quad \boldsymbol{A}$, Target RDM reflecting a theoretical three-factor structure clustering the self, social network members (close others and acquaintances), and celebrities. $\boldsymbol{B}, \mathrm{A}$ whole-brain searchlight RSA revealed brain regions whose similarity structures reflected this target structure (voxelwise threshold, $p<0.001$; cluster-corrected, $p<0.001$ ).

for a linear increase in neural response as a function of target category (i. e., self, close others, acquaintances, celebrities) in a linear mixed-effects model with a random intercept for subject; and (2) regressed neural responses with self-other closeness ratings for each target in a linear mixed-effects model with a random intercept for subject and target, using the lme4 (Bates et al., 2015) and lmerTest (Kuznetsova et al., 2017) packages in R. In addition, we completed follow-up, whole-brain analyses testing for regions across the brain whose activation amplitude and multivariate pattern similarity were modulated by self-other closeness. We report Satterthwaite approximated degrees of freedom for the linear mixed-effects models.

Univariate analyses assessing the impact of self-other closeness. First, activation betas associated with each target were extracted from the mPFC ROI and were regressed with (1) target category (self $>$ close others $>$ acquaintances $>$ celebrities) and (2) subjective measures of self-other closeness for each target. Second, a whole-brain parametric modulation analysis was conducted to identify regions of the brain whose activation magnitude increased with self-other closeness. A subject-level regressor reflecting the closeness ratings for each target (from 1 to 100, with closeness to the self defined as 100) were entered into the first-level GLM to identify brain regions whose activity linearly increased with self-other

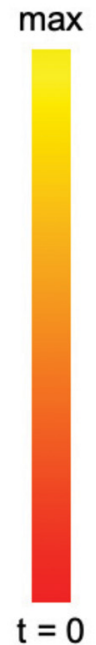

closeness. Next, a second-level one-sample $t$ test was conducted, and the resulting group-level map was voxelwise-thresholded at $p<0.001$ and cluster-corrected to $p<0.001$ (minimum extent threshold: $k=66$ contiguous voxels), as recommended by AFNI's 3dClustSim, using the spatial autocorrelation function (see Eklund et al., 2016; Cox et al., 2017).

Multivariate analyses assessing the impact of self-other closeness. Multivariate responses in the mPFC ROI were extracted for a comparison of activation patterns between the self and others, across targets. The similarity (Fisher $z$ transformed correlation value) between each target and the self was then regressed with (1) target category (close others $>$ acquaintances $>$ celebrities) and (2) self-other closeness ratings for each target. To determine whether additional brain regions demonstrate increases in neural similarity between the self and others with greater closeness, a sphere searchlight ( $3 \mathrm{~mm}$ radius) was used to conduct a wholebrain RSA. Specifically, this model assessed similarity in multivariate responses between the self condition and each other target, weighted by the subject's closeness rating for that target (i.e., comparing neural patterns to the self with each target, weighted by each subject's vector of 15 closeness ratings). The 1-100 closeness ratings were transformed to a $0-1$ scale and converted to distances ( 1 - the transformed value) to permit correlation with neural RDMs. Similarity in activation patterns was estimated using Pearson correlation and similarity across brain activation patterns and behavioral responses were estimated using Spearman rank correlation. These correlation values were Fisher $z$-transformed to allow for statistical comparisons between target categories.

Relating self-other neural responses in MPFC and PCC to loneliness. Next, we sought to determine whether loneliness alters the representation of subjective social connections in mPFC, as well as PCC. We examined two possibilities: (1) whether loneliness alters the mapping of social circles in mPFC and PCC and (2) whether loneliness is associated with greater dissimilarity in representations between the self and others in $\mathrm{MPFC}$ and PCC. To test the first possibility that loneliness may be associated with altered mapping of social circles, we extracted pairwise neural similarity (Fisher $z$-transformed correlation coefficients) in each of these ROIs to examine within-category similarity for each social circle (i.e., close others, acquaintances, and celebrities) and between-category similarity as a function of the distance between the social circles, according to their closeness to the self (i.e., at each step from the diagonal of the RDM). This revealed neural similarity for targets within a category (e.g., how similarly close others are represented to one another, how similarly acquaintances are represented to one another, and how similarly celebrities are represented to one another; distance of 0 ), from adjacent target categories (e.g., close others and acquaintances, and separately, acquaintances and celebrities; distance of 1), and from distant target categories (close others and celebrities; distance of 2). In these models, pairwise neural similarity among targets was predicted by both loneliness and either (1) the distance between target categories (or social circles) in the RDM or (2) the particular target category (for targets in the same social circle). A median split on loneliness (median $=43$ ) was conducted for visualization and for examining simple effects. All other models used continuous 
loneliness values. Full models predicting similarity from loneliness and target category or between-target category distance included a random intercept for subject. To avoid overfitting, models testing simple effects of loneliness on neural similarity for a particular target category or between-category distance included a random intercept for the particular target pair (e.g., close 1-close 2).

Finally, to test the second possibility that loneliness is associated with greater dissimilarity in representations between the self and others in mPFC and PCC, participants' loneliness scores were entered as a predictor of univariate neural activation and multivariate self-other neural similarity (Fisher $z$-transformed correlation coefficients of each category with the self) in each of these ROIs, along with either target category or self-other closeness ratings. Specifically, separate regression models predicted activation magnitude in either of these regions as a function of: (1) target category, linearly ordered by closeness to the self (self $>$ close others $>$ acquaintances $>$ celebrities); or (2) subjective self-other closeness ratings to each target. Similarly, separate regression models predicted self-other multivariate similarity in these regions as a function of: (1) target category (close others $>$ acquaintances $>$ celebrities) or (2) subjective self-other closeness ratings. The models with self-other closeness as a predictor included a random intercept for target; to avoid overfitting, those with target category as a predictor did not.

\section{Results}

Does mPFC maintain an organized map of ourselves and our social circles?

Social category representation in $\mathrm{MPFC}$

To interrogate the structure of self-other representation in the mPFC, we extracted the neural RDM from the mPFC ROI (Fig. $2 A$ ). The overall representational structure in the mPFC differentiated the self from all other target categories, whereas the representation of others was clustered such that all social network members (close others and acquaintances) were similarly represented to one another and celebrities were, separately, similarly represented to one another (Fig. $2 B$ ). To better visualize the emergent structure of the matrix, a multidimensional scaling solution was derived to depict the similarity of target categories along two dimensions (Fig. 2C). This plot more clearly illustrates that three clusters emerge from cross-category similarity in mPFC activation: self, social network members (close others and acquaintances), and celebrities. Indeed, the neural RDM from this region correlated with this "three-cluster model" $(r=0.34$, $\left.t_{(42)}=10.78, p<0.001\right)$. To further confirm that mPFC adheres to this three-cluster model of self and other representation, we submitted the average distance matrix yielded by the RSA in the mPFC to a common $k$-medoids clustering technique, partitioning around medoids, which is known to perform well with distance matrices (Van der Laan et al., 2003). Specifically, we tested a variety of clustering solutions to identify the one that best describes the data, using the silhouette width, a metric that weighs the distance between points in different clusters to those within the same cluster. Higher silhouette widths (ranging from 0 to 1 ) indicate more separability of the clusters. Thus, a clustering solution (i.e., number of clusters) that maximizes the silhouette width (or distance between clusters) suggests a better model fit. Of all possible clustering solutions, the three-cluster model yielded the maximum average silhouette width (0.24) and best fit for our data. Moreover, it maintained the most meaningful solution, with all targets falling within expected clusters. Collectively, these results suggest that the mPFC adheres to a three-cluster model of self and other representation, with representations of self, social network members (close others and acquaintances), and celebrities separately clustered.
Table 1. Peak regions from searchlight analysis for three-factor structure clustering the self, social network members (close others and acquaintances), and celebrities (voxelwise threshold, $p<0.001$; cluster-corrected, $p<0.001)^{\mathrm{a}}$

\begin{tabular}{lrrrlr}
\hline & \multicolumn{3}{l}{ Coordinates (MNI) } & & \\
\cline { 2 - 4 } Region & \multicolumn{1}{c}{$x$} & \multicolumn{1}{c}{$y$} & \multicolumn{1}{c}{$z$} & Volume $\left(\mathrm{mm}^{3}\right)$ & Peak $T$ \\
\hline PCC/precuneus & -6 & -54 & 30 & 34137 & 16.19 \\
Precuneus & 0 & -66 & 27 & & 13.77 \\
mPFC & 0 & 45 & -9 & & 12.85 \\
MTG & 57 & -6 & -27 & & 10.48 \\
Right TPJ & 51 & -60 & 18 & & 9.69 \\
Caudate tail & 24 & -27 & 21 & 257 & -7.27 \\
Midbrain & 0 & -15 & -6 & 249 & -6.12 \\
\hline
\end{tabular}

${ }^{a}$ MTG, Middle temporal gyrus; rTPJ, right temporoparietal junction. Volumes refer to entire suprathreshold clusters. Cluster peaks and selected subpeaks are indicated by their region names (adapted from Automated Anatomical Labeling in SPM).

\section{Social category representation across the whole brain}

To search for other brain regions with representational profiles similar to the one reflected in the mPFC, a whole-brain searchlight analysis was performed using the three-cluster, social category structure as the target RDM (Fig. 3A). The results of this analysis were not meant to confirm findings from the mPFC ROI, per se, as such an approach would be susceptible to issues surrounding nonindependence (i.e., "double-dipping") (Poldrack and Mumford, 2009; Vul et al., 2009). Instead, this analysis helps identify additional brain regions that similarly cluster these target conditions. Indeed, this analysis yielded regions across the social brain (voxelwise threshold, $p<0.001$; cluster-corrected, $p<0.001$; Table 1). In addition to $\mathrm{mPFC}$, this analysis revealed a cluster in PCC/precuneus that extended into temporoparietal junction, middle temporal gyrus, and temporal poles (Fig. 3B). Thus, in addition to the $\mathrm{mPFC}$, multiple additional brain regions implicated in social cognition adhere to the three-cluster model of self and other representation, with representations of self, social network members (close others and acquaintances), and celebrities separately clustered. We note that the whole-brain correlation map resulting from a searchlight with a four-cluster target structure, mirroring the target categories (self, close others, acquaintances, and celebrities) from the experimental design, revealed a similar set of brain regions to the three-cluster structure.

\section{Does subjective self-other closeness modulate similarity in mPFC responses to self and others?}

Neural responses to self-other closeness in $M P F C$

To isolate the influence of social closeness on neural responses to the self and others, we began by assessing whether univariate response magnitudes in the $\mathrm{mPFC}$ were sensitive to the participant's closeness to each target. Consistent with this prediction, activation magnitudes in the a priori-defined mPFC ROI linearly increased with the social closeness of the target to the participant (linear effect $\times$ target condition: $B=0.33, t_{(642)}=8.59, p<$ 0.001 ; linear increase with social closeness ratings: $B=0.13, t_{(29.3)}$ $=5.67, p<0.001$; Fig. $4 A$ ). This result complements and extends previous literature demonstrating intermediate activation of the mPFC for a single close other relative to the self and a single distant other (Krienen et al., 2010; Feng et al., 2018).

Parametric modulation by self-other closeness across whole brain Next, we conducted a whole-brain parametric modulation analysis to more extensively search for brain regions that were sensitive to the closeness of the target considered during the task. This analysis revealed a single cluster in the $\mathrm{MPFC}$ (MNI: $-3,33,-9$; 
A

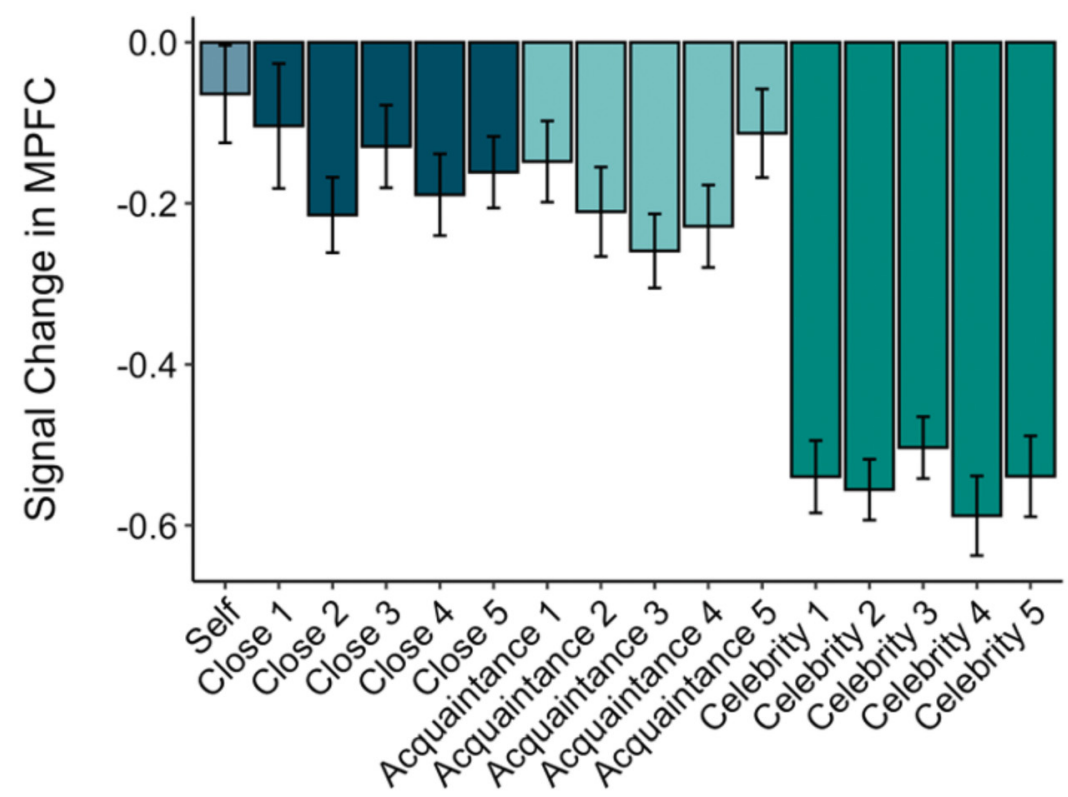

B
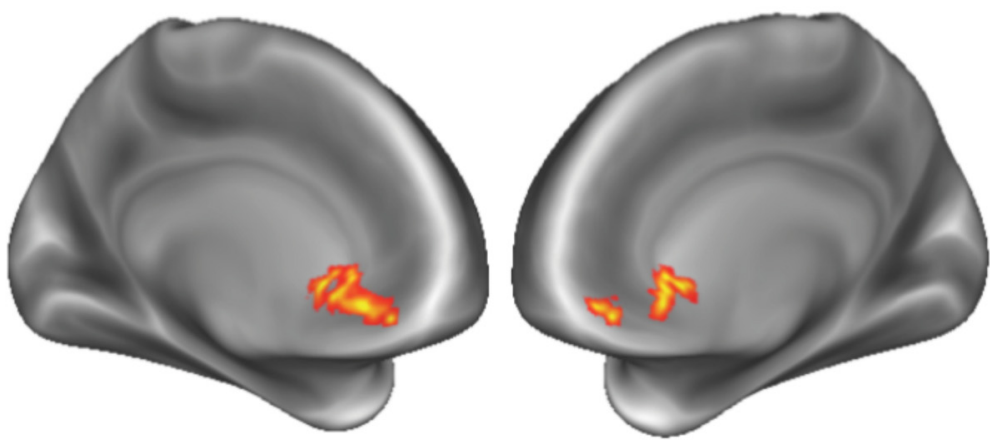

Figure 4. A, Activation magnitude in the $\mathrm{mPFC} R 0 \mathrm{l}$ linearly increased with self-other closeness (linear effect $\times$ target condition: $B=0.33, t_{(642)}=8.59, p<0.001$; linear increase with subjective closeness ratings: $B=0.13, t_{(29.3)}=5.67, p<$ 0.001). Error bars represent standard errors. $B$, Whole-brain parametric modulation analysis revealed a single cluster in the $\mathrm{mPFC}(-3,33,-9)$ whose activation magnitude linearly increased with the self-other closeness of the participant and target (voxelwise, $p<0.001$; cluster-corrected, $p<0.001$ ).

voxelwise, $p<0.001$; cluster-corrected, $p<0.001)$ whose BOLD activation linearly increased with the social closeness of the target to the participant (Fig. 4B). Both univariate analyses suggested that activation in the $\mathrm{mPFC}$, defined $a$ priori for its association with self-related processing and identified whole brain, is modulated by social closeness.

\section{Neural representation of self-other closeness in $m P F C$}

To determine whether multivariate activation patterns in the mPFC were sensitive to subjective self-other closeness, we extracted parameter estimates that reflected the similarity of each target to the self in this region. Self-other overlap did not linearly increase with target category $\left(B=0.03, t_{(600)}=\right.$ $1.43, p=0.15)$, and only marginally increased as a function of target-specific self-other closeness ratings $\left(B=0.02, t_{(18.4)}\right.$ $=1.96, p=0.066)$. However, we found evidence for greater neural self-other overlap with close others compared with acquaintances and celebrities. That is, the direct comparison $\max$

of neural self-other overlap with close others relative to both acquaintances and celebrities was significant $(B=0.06$, $t_{(600)}=2.70, p=0.007$; Fig. 5). These findings provide novel and rigorous support for the social psychological idea that close others are incorporated into our own self-representations (Aron et al., 1991).

\section{Searchlight analysis for self-other closeness}

For a more extensive exploration of how social closeness moduates self-other neural similarity, we conducted a whole-brain searchlight analysis on self-other closeness ratings. That is, closeness ratings were used as the comparison metric (i.e., target similarity metric) for self-other neural similarity. This analysis revealed regions where targets who are personally close to the self also elicit similar activation patterns as the self. This searchlight analysis revealed regions across the social brain, including the PCC/precuneus and mPFC, whose patterns of self-other similarity best matched the subjective closeness ratings reported by the participants (voxelwise threshold, $p<0.001$; clustercorrected, $p<0.001$; Table 2; Fig. 6). Although the peak of this cluster is more dorsal than the mPFC ROI, the cluster extent appearing at a slightly less conservative threshold (voxelwise, $p<0.005$; cluster extent, $p<0.005$ ) overlaps extensively with the voxels in the mPFC ROI.

Overall, it appears that the magnitude of neural activity and multivariate representation in $\mathrm{mPFC}$ in response to other targets become increasingly similar to the self with subjective social closeness.

\section{Is loneliness associated with altered responses to the self and others in mPFC and PCC?}

To test the possibility that neural responses to the self and others could reflect meaningful individual differences in social connection, we related both univariate and multivariate parameter estimates in the MPFC and PCC ROIs to trait loneliness. These regions were selected for their association with both social category and selfother closeness representation in the previous whole-brain analyses. These ROIs were defined independently of our own data (see Materials and Methods) for the following analyses.

\section{Loneliness modulates univariate activation to the self and others} in $m P F C$

Examining neural activation magnitude in mPFC across all target categories revealed a main effect (ME) of loneliness whereby greater loneliness was associated with less mPFC activation $(B=$ $\left.-0.065, t_{(648)}=-3.43, p<0.001\right)$; this effect did not differ across target categories (interaction between linear trend for target category and loneliness: $B=0.055, t_{(648)}=1.24, p=0.22$ ). In a similar 
model of mPFC activation by self-other closeness and loneliness, there was a ME of loneliness whereby greater loneliness was associated with less $\mathrm{mPFC}$ activation $\left(B=-0.032, t_{(645.8)}=-2.12, p=0.035\right)$; this effect did not differ as a function of self-other closeness (interaction of selfother closeness and loneliness: $B=0.016$, $\left.t_{(638.9)}=1.02, p=0.31\right)$. That is, lonelier individuals may exhibit less mPFC activity while reflecting on people, regardless of the person considered. In contrast, loneliness was not associated with neural activation in the PCC (ME loneliness in model with condition: $B=-0.011, t_{(648)}=-0.53$, $p=0.59$; ME loneliness in model with selfother closeness: $B=-0.003, t_{(646.6)}=$ $-0.19, p=0.85)$ and did not differ across target categories $\left(B=0.01, t_{(648)}=0.19\right.$, $p=0.85)$ or as a function of self-other closeness $\left(B=0.014, t_{(638.1)}=0.78, p=\right.$ $0.44)$.

Loneliness modulates representation of social circles in $\mathrm{MPFC}$ and PCC

To test whether loneliness modulates the mapping of social circles in mPFC and PCC, we first compared neural (dis)similarity of each pair of targets, excluding the self condition, as a function of their distance from each other relative to the self (i.e., at each step from the diagonal of the similarity matrix). This analysis assesses how similarly targets within a category were represented (distance of 0 ), how similarly targets from adjacent categories were represented to one another (close others and acquaintances, and separately, acquaintances and celebrities (distance of 1), and how similarly the two most distant categories, close others and celebrities, were represented to one another (distance of 2). Moreover, we relate within and between-category similarity to self-reported trait loneliness.

Overall, we observed a decay in the neural similarity of social targets with increasing distance between the social categories in $\mathrm{mPFC}$ and PCC. Within-category similarity (distance of 0 ) was higher than the similarity between adjacent categories (i.e., distance of 1: close others and acquaintances; acquaintances and celebrities $)$ in both $\operatorname{mPFC}\left(B=0.16, t_{(4262)}=13.355, p<0.001\right)$ and PCC $\left(B=0.21, t_{(4262)}=17.66, p<0.001\right)$. In turn, similarity between adjacent categories was higher than the similarity between distal categories (distance of 2: close others and celebrities $)$ in $\operatorname{mPFC}\left(B=0.07, t_{(4262)}=5.35, p<0.001\right)$ and PCC $(B=$ $\left.0.16, t_{(4262)}=12.95, p<0.001\right)$. Critically, loneliness moderated the similarity within and between these social circles (Fig. $7 B$ ). More specifically, as loneliness increased, adjacent target categories (i.e., distance of 1: close others and acquaintances; acquaintances and celebrities) were represented more similarly to one another in both the $\operatorname{mPFC}\left(B=0.004, t_{(1999)}=4.87, p<0.001\right)$ and PCC $\left(B=0.007, t_{(1999)}=7.91, p<0.001\right.$; Fig. $\left.7 A, B\right)$. In $\mathrm{mPFC}$, this blurring of social circles with loneliness was even observed in the similarity between close others and celebrities (i.e., distance of $2 ; B=0.003, t_{(999)}=2.40, p=0.017$; Fig. $7 A$ ).

In addition to changes between social circles, loneliness may alter within social circle mapping as well. To explore this possibility, we examined pairwise neural similarity for social targets belonging to the same social circle. Most strikingly, as loneliness
Table 2. Peak regions from searchlight analysis comparing self-other neural similarity to social closeness ratings (voxelwise threshold, $p<0.001$; clustercorrected, $p<0.001)^{\mathrm{a}}$

\begin{tabular}{|c|c|c|c|c|c|}
\hline \multirow[b]{2}{*}{ Region } & \multicolumn{3}{|c|}{ Coordinates (MNI) } & \multirow[b]{2}{*}{ Volume $\left(\mathrm{mm}^{3}\right)$} & \multirow[b]{2}{*}{ Peak $T$} \\
\hline & $x$ & $y$ & $z$ & & \\
\hline $\mathrm{mPFC}$ & 9 & 51 & 33 & 235 & 6.43 \\
\hline Dorsal mPFC & 12 & 39 & 45 & & 5.27 \\
\hline Right SFG & 21 & 33 & 42 & & 3.93 \\
\hline $\mathrm{PCC} /$ precuneus & -6 & -24 & 39 & 659 & 6.51 \\
\hline Mid-cingulate & -3 & -15 & 39 & & 6.27 \\
\hline Mid-cingulate & 3 & -24 & 42 & & 6.04 \\
\hline Visual cortex & -6 & -90 & -3 & 2957 & 12.34 \\
\hline Lingual gyrus & 6 & -84 & -3 & & 11.67 \\
\hline Lingual gyrus & 15 & -84 & -6 & & 11.61 \\
\hline Left SFG & -24 & 36 & 45 & 260 & 6.40 \\
\hline Left SFC & -24 & 27 & 42 & & 5.31 \\
\hline Left MFG & -33 & 24 & 48 & & 4.91 \\
\hline
\end{tabular}

${ }^{a_{S}} \mathrm{SFG}$, Superior frontal gyrus; SFC, superior frontal sulcus; MFG, middle frontal gyrus. Volumes refer to entire suprathreshold clusters. Cluster peaks and selected subpeaks are indicated by their region names (adapted from Automated Anatomical Labeling in SPM).

increased, acquaintances were represented more similarly to one another in the $\operatorname{mPFC}\left(B=0.004, t_{(399)}=1.98, p=0.049\right.$; Fig. $\left.7 C\right)$ and PCC $\left(B=0.008, t_{(399)}=3.96, p<0.001\right.$; Fig. $\left.7 D\right)$. Close others $\left(B=0.008, t_{(399)}=3.73, p<0.001\right)$ and celebrities $(B=$ $\left.0.008, t_{(399)}=3.85, p<0.001\right)$ were also represented more similarly to one another in PCC (Fig. 7D).

Collectively, these results suggest that loneliness is associated with an altered neural map of social circles. Although there were some differences in this alteration between mPFC and PCC, in both of these regions, loneliness was associated with blurred boundaries between the social circles surrounding acquaintances (i.e., increased similarity between close others and acquaintances, as well as acquaintances and celebrities), and blurred boundaries among the collection of acquaintances themselves (i.e., increased 


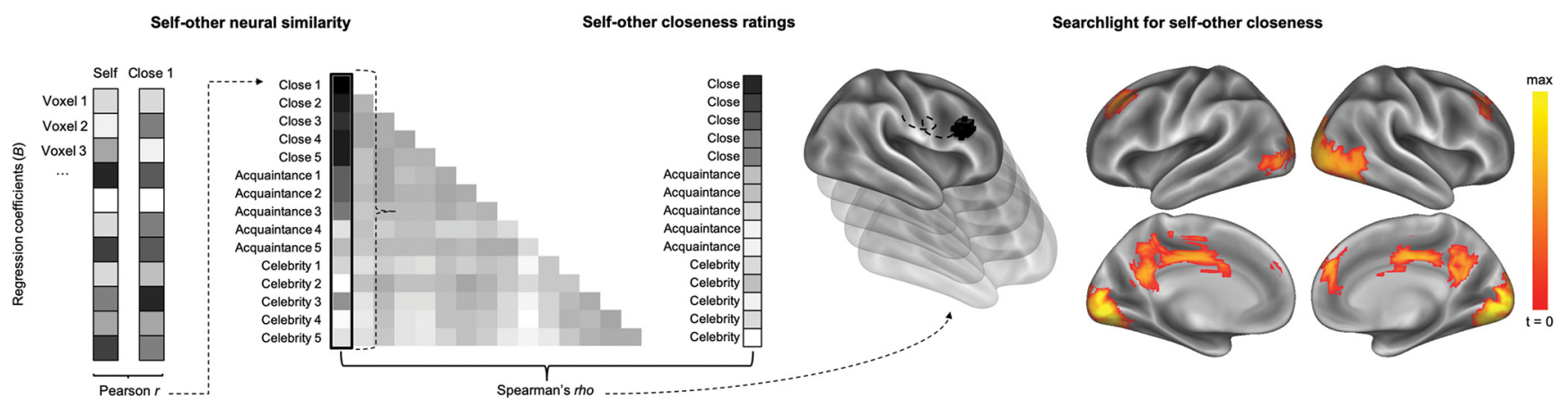

Figure 6. A whole-brain searchlight RSA for self-other closeness. Brain regions where self-other neural similarity was positively correlated with ratings of self-other closeness included the $\mathrm{PCC} /$ precuneus and $\mathrm{mPFC}$ (voxelwise threshold, $p<0.001$; cluster-corrected, $p<0.001$ ).

similarity between acquaintances). Notably, this cannot be explained by a difference in perceived closeness to the targets for lonely participants, as both those low and high in loneliness report greater closeness to close friends than acquaintances (low loneliness: $B=26.91, t_{(215)}=20.46, p<0.001$; high loneliness: $B$ $\left.=25.13, t_{(215)}=18.84, p<0.001\right)$ and to acquaintances than celebrities (low loneliness: $B=30.08, t_{(215)}=23.86, p<0.001$; high loneliness: $\left.B=24.42, t_{(215)}=17.86, p<0.001\right)$.

\section{Loneliness modulates self-other similarity in $\mathrm{MPFC}$ and PCC}

Finally, to test the possibility that loneliness is associated with a disconnected neural self-representation, we regressed self-other similarity in multivariate activation patterns with the loneliness of the participant, separately for the $\mathrm{mPFC}$ and PCC ROIs. In the $\mathrm{MPFC}$, loneliness negatively related to self-other similarity across all target categories (ME loneliness in model with condition: $B=-0.05, t_{(609)}=-3.91, p<0.001$; ME loneliness in model with self-other closeness: $B=-0.05, t_{(605.6)}=-3.67, p<$ $0.001)$; and this did not differ as a function of target category $\left(B=0.008, t_{(609)}=0.33, p=0.74\right.$; Fig. $\left.8 A\right)$ or self-other closeness $\left(B=-0.003, t_{(597.7)}=-0.19, p=0.85\right)$. Conversely, in the PCC, loneliness positively related to self-other similarity across all target categories (ME loneliness in model with condition: $B=0.073$, $t_{(609)}=5.17, p<0.001 ;$ ME loneliness in model with self-other closeness: $\left.B=0.08, t_{(611)}=5.46, p<0.001\right)$; and this did not differ as a function of target category $\left(B=-0.02, t_{(609)}=-0.82, p=\right.$ 0.41 ; Fig. $8 B)$ or self-other closeness $\left(B=-0.002, t_{(611)}=-0.13\right.$, $p=0.90)$. These results suggest that lonelier individuals may indeed represent others as more distant or dissimilar from the self in the mPFC. In contrast, lonelier individuals may represent themselves as more connected or similar to others in the PCC.

\section{Discussion}

How does the brain represent our subjective social connection to others? Here, we found that multivariate activation patterns in the $\mathrm{mPFC}$ and regions across the social brain clustered targets by social category. Specifically, the social brain (1) represented the self distinctly from others and (2) clustered the representation of others based on whether or not they belong to our social network. Although the self was represented distinctly from others, self-other closeness modulated this distance. Greater subjective self-other closeness was associated not only with greater response amplitudes in $\mathrm{mPFC}$, but also more similar neural activation patterns in both mPFC and throughout the social brain. Critically, loneliness moderated the organization of the self and others, with blurred representations of weaker ties (i.e., acquaintances), and altered neural self-other overlap. Collectively, these results provide novel insight into how the social brain maps our subjective connections to others, and how loneliness alters this mapping.

Most people maintain a core set of 3-5 close others but regularly interact with larger groups of friends and acquaintances, with diminishing frequency and emotional attachment for more peripheral relationships (Zhou et al., 2005; Roberts and Dunbar, 2011; Dunbar, 2018). Indeed, it is thought that the time, effort, financial, and even information processing costs of maintaining relationships constrain the size of each of these social network layers (Stiller and Dunbar, 2007; Roberts and Dunbar, 2011). Thus, both the broad clustering of individuals based on whether or not they are in our social network (close others and acquaintances vs celebrities) and the finer-grained increase in self-other overlap with interpersonal closeness may help us efficiently respond to individuals in our social environment. To further test this possibility, future work may examine whether individuals with the largest social networks also have the most efficiently organized representations between the self and others. Just as clearer physical maps help us navigate physical space, clearer interpersonal maps may help us navigate social interactions.

Self-other closeness increases neural representational overlap To our knowledge, our results provide the most rigorous assessment to date of increasing neural self-other overlap with interpersonal closeness, finding evidence for self-other overlap in mPFC, as well as PCC. Self-other overlap is an important construct in social psychology: it is a defining feature of interpersonal relationships (Aron et al., 1991; Branand et al., 2019) and corresponds with prosocial outcomes, such as enhanced empathy (Galinsky et al., 2005). Yet, it has been difficult to precisely test for overlapping representations between the self and others, in part due to methodological limitations. Self-other overlap is typically assessed by (1) asking participants to indicate the degree of overlap they feel they share with others (Aron et al., 1991), (2) source errors between the self and others in memory (Benoit et al., 2010; Bergström et al., 2015), and/or (3) comparing univariate activity in mPFC to the self and a single close other and non-close other (Krienen et al., 2010). Given that none of these methods assesses representational content, evidence for selfother overlap from these measures cannot rule out alternative interpretations. We capitalized on multivariate neural pattern similarity to more precisely demonstrate overlapping representations between the self and others as a function of closeness. Building on prior literature (Schmitz and Johnson, 2007), we suggest that the blurring of self-other representations observed here could be due to the interpersonal nature of the self, the 


\section{Between Category Neural Similarity}

A

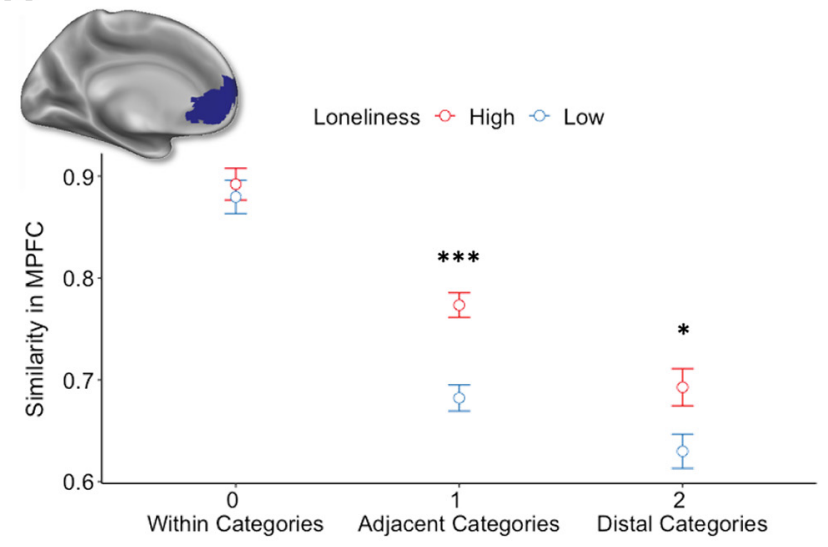

Distance betweeen categories
B

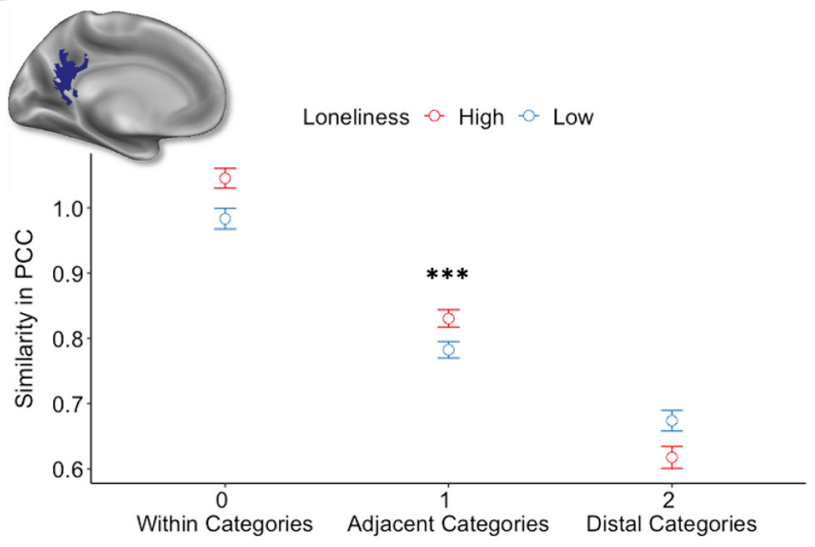

Distance between categories

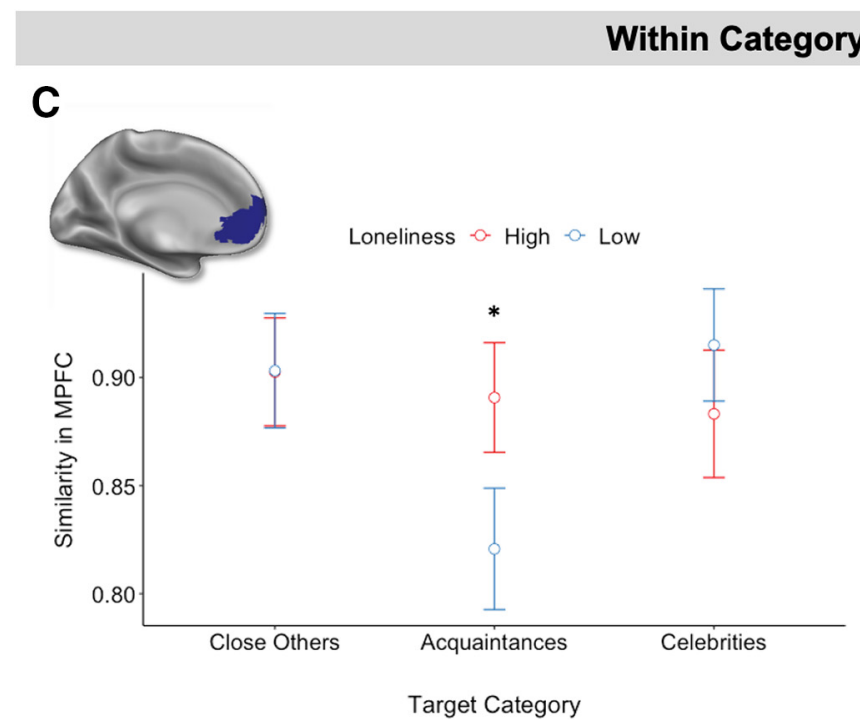

\section{Neural Similarity}

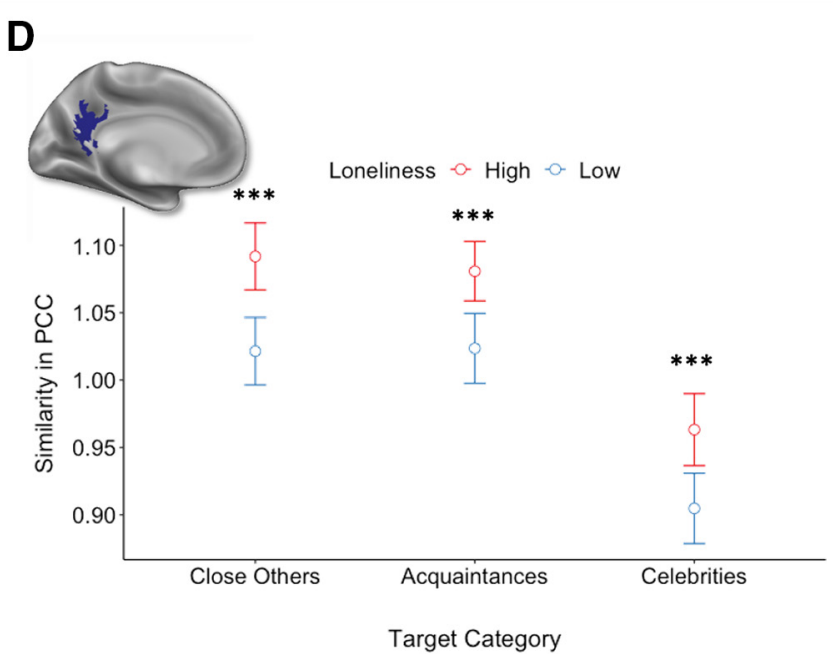

Figure 7. A, Between-category neural similarity in mPFC decreased with the distance between target categories relative to the self. $\boldsymbol{B}$, Similarly, between-category neural similarity in PCC decreased with social distance. C, Within-category neural similarity in $\mathrm{mPFC}$ for high and low lonely individuals. $\boldsymbol{D}$, Within-category neural similarity in PCC for high and low lonely individuals. Loneliness data were median split for visualization but used continuously during analysis. Error bars represent standard errors. Significance of the simple effects: ${ }^{*} p<0.05 ;{ }^{* * *} p<0.001$.

richness with which personally known others (including the self) are represented, and/or the heightened subjective value associated with relationship partners.

For example, some have suggested that the self can only be defined in the context of its relationship to others (Andersen and Chen, 2002). As such, a latent, relational version of the self may exist for each unique relationship, which comprises all aspects of the self that are most relevant for that relationship (Andersen and Chen, 2002). Thus, neural self-other overlap may reflect the relational self associated with the target considered. Alternatively, the increase in neural self-other similarity with subjective closeness may reflect more shared experience with these individuals, as a shared history might provide more information to enrich mental models of the person (Schmitz and Johnson, 2007; Murray et al., 2012). Consistent with this possibility, the more experience we have with a person, the richer our multivariate $\mathrm{mPFC}$ representation of their mental states (Thornton et al., 2019).

Additionally, social network members may be endowed with enhanced subjective value, which is frequently associated with the ventral mPFC (Wagner et al., 2019). Indeed, the self and subjective value may be intrinsically related (Tamir and Mitchell, 2012; Chavez et al., 2017) and associated with closeness to the self (Schmitz and Johnson, 2007; Murray et al., 2012). Interestingly, the parametric modulation analysis, which tested for neural activity that increased with closeness to the self, yielded a ventral portion of the MPFC similarly located to clusters associated with subjective value. (Bartra et al., 2013; Clithero and Rangel, 2014). In contrast, the cluster that emerged in the multivariate self-other overlap analysis was more dorsal, and is a region frequently associated with person perception and social inference (Lieberman et al., 2019). Mean levels of neural activity in vmPFC may track the value of others, whereas shared multivariate responses in more dorsal portions of $\mathrm{mPFC}$ may reflect shared representational content between the self and others.

Surprisingly, although the visual characteristics of the stimuli did not differ across conditions, activation patterns in the visual cortex were increasingly similar for targets that were closer to the self. One possibility is that these responses may be driven by the increased salience of closer targets. Previous research has demonstrated increased activation in the visual cortex during judgments 


\section{A}
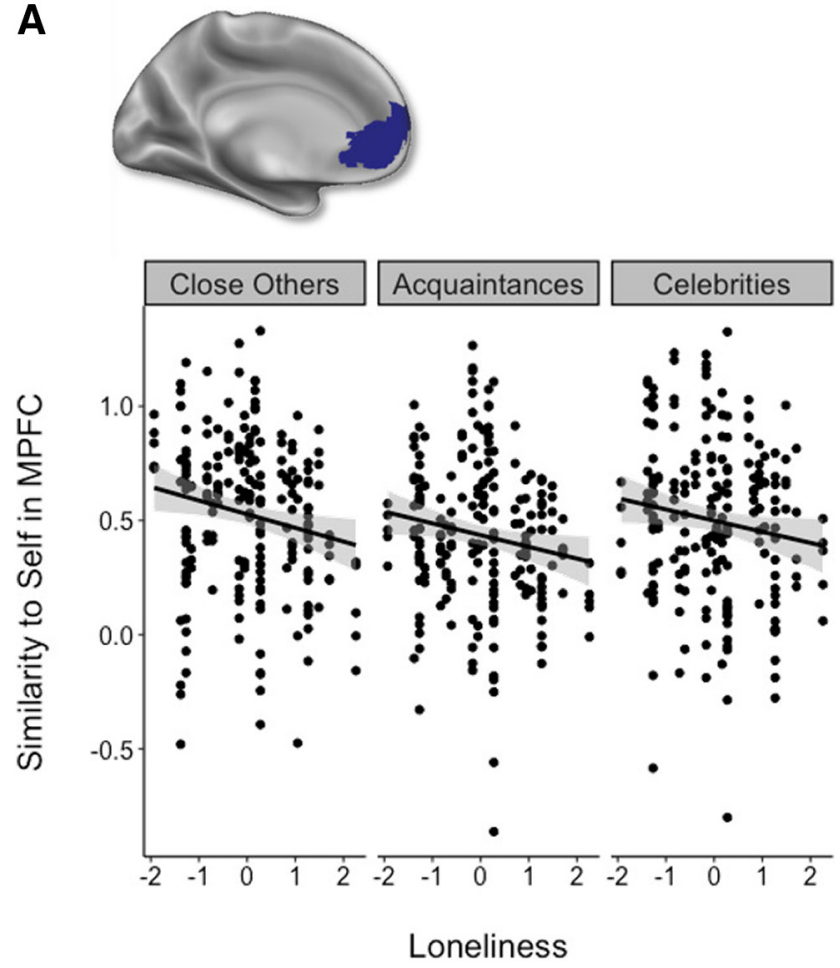

B
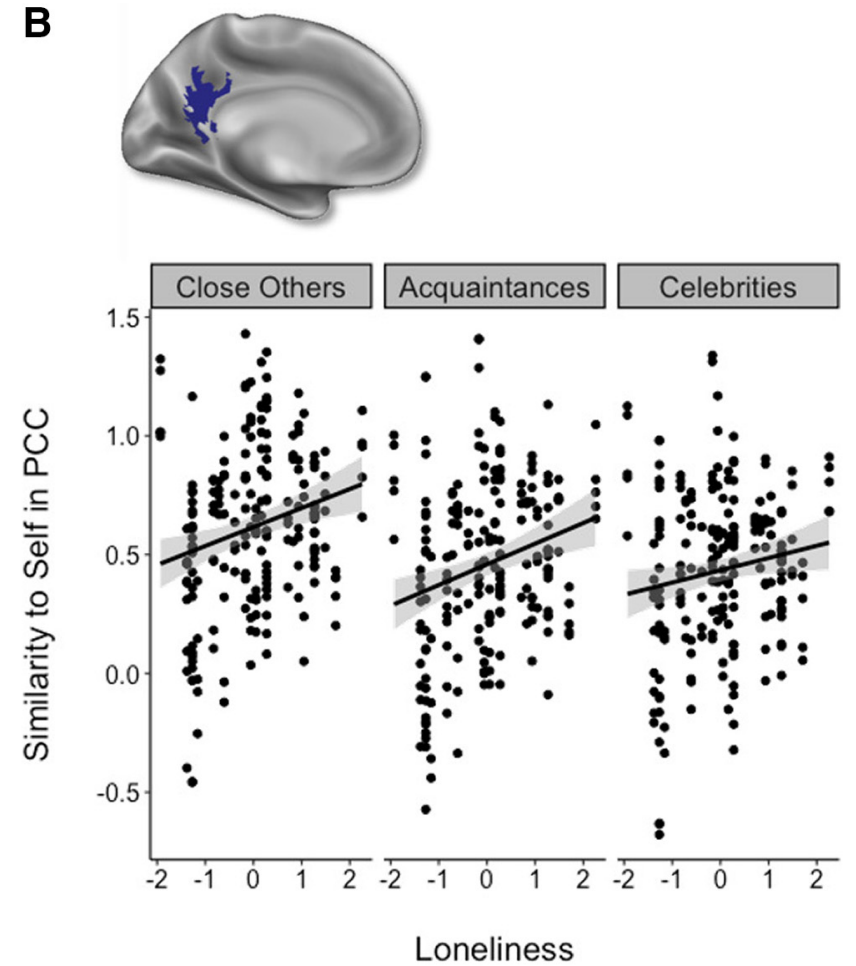

Figure 8. $\boldsymbol{A}$, Loneliness was associated with lower self-other similarity in the mPFC across all target categories $\left(B=-0.05, t_{(609)}=-3.91, p<0.001\right)$. $\boldsymbol{B}$, Conversely, loneliness was associated with greater self-other similarity in the PCC across all target categories $\left(B=0.073, t_{(609)}=5.17, p<0.001\right)$. Confidence bands reflect $95 \%$ confidence intervals.

of moral emotions (e.g., embarrassment and guilt) from written text (Takahashi et al., 2004). Our task was similar to this past work in that it required judgments of personality characteristics for multiple targets; and these judgments may have been more strongly evoked for targets who are more emotionally close to the participant. Moreover, neural signal in the visual cortex is less susceptible to variation across individuals than the PFC (Chang et al., 2018), which might allow for stronger correlations across conditions and greater detection at the whole-brain level.

\section{Loneliness alters neural self-other representation}

Our findings also offer novel insight into the brain basis of loneliness. Loneliness has been defined as the perception of isolation, with the perceived gap between the self and others a defining feature of loneliness phenomenology (Hawkley and Cacioppo, 2010). Indeed, standard measures of loneliness point to a selfrepresentation that is disconnected from others in the environment. The reliable, valid, and heavily used questions on the UCLA Loneliness Scale characterize participants' loneliness, in part, by their responses to the following questions: "I feel disconnected from others"; "people are around me but not with me"; and "my interests and ideas are not shared by those around me" (Russell et al., 1980). Yet, to date, neuroscience studies on loneliness focus on how loneliness modulates responses to social cues in the environment (Cacioppo et al., 2009), but not on the perceived gap between self and others. For example, lonelier individuals show less reward system activation in response to strangers (Cacioppo et al., 2009), more reward system activity in response to close others (Inagaki et al., 2016), and exaggerated visual attention to negative social cues (Cacioppo et al., 2009). While this past research is important for understanding the socio-affective responses tied to loneliness, it does not speak to the brain basis of the mental representation of subjective isolation. The observation that, in $\mathrm{mPFC}$, a region consistently implicated in person representation (Denny et al., 2012; Wagner et al., 2012), loneliness corresponded with self-representations that are more dissimilar from representations of others provides the first neural account of the perceived self-other disconnection associated with loneliness. Specifically, our results suggest that the subjective experience of loneliness can be traced to a lonelier "neural self," with lonelier individuals distancing themselves from their social connections, even at the level of neural representation.

Our approach also identified an association between loneliness and greater similarity between the self and others in PCC. The PCC has been shown to dynamically track qualities of interpersonal relationships between self and others (Tavares et al., 2015). For example, the PCC keeps track of, in terms of the absolute difference between the self and other, the difference in power and affiliation between the self and other in the relationship (e.g., a relationship with a greater absolute difference in power would indicate less power balance between relationship partners) (Tavares et al., 2015). Here this was reflected in greater similarity between the self and close others in PCC. Moreover, the increased similarity between the self and others observed in the PCC at higher levels of loneliness may suggest a failure to distinguish the nuances between self and others in interpersonal space, which may contribute to and/or be a consequence of lonelier individuals' difficulties navigating interpersonal interactions (Jones et al., 1982; Wittenberg and Reis, 1986; Spitzberg and Hurt, 1987; Segrin and Flora, 2000).

Finally, lonelier participants had blurred boundaries in mPFC and PCC between the social circles surrounding acquaintances, as well as blurred boundaries among the collection of acquaintances themselves. Moreover, this was true despite the fact that individuals high and low in loneliness report feeling closer to close others than acquaintances, and closer to acquaintances than celebrities. Chronic social disconnection may therefore 
suppress the distinctiveness with which peripheral social network members are represented in both of these brain regions. Interestingly, social network research emphasizes the "strength of weak ties," specifically highlighting the important role acquaintances play in well-being, social support, and access to information (Granovetter, 1973; Wellman and Wortley, 1990; Sandstrom and Dunn, 2014). However, neuroscience research on loneliness focuses on lonely individuals' responses to either strangers (Cacioppo et al., 2009; Yamada and Decety, 2009) or close others (Inagaki et al., 2016), but not acquaintances. Our findings suggest that understanding how the brain represents weak ties may yield important insight into how loneliness links to negative outcomes.

In conclusion, the quality and intimacy of social relationships are critical predictors of happiness and well-being (Klinger, 1977; Diener and Seligman, 2002; Holt-Lunstad et al., 2010). Our results suggest that the social brain may help us navigate our social connections by mapping people based on whether or not they are in our social network, with our closest social ties represented most closely to ourselves. Moreover, loneliness is associated with distortions in this mapping, particularly skewed neural similarity between the self and others and blurred representations of weak ties. The paths we take in social life may depend, in part, on the interpersonal maps we carry in our social brains.

\section{References}

Andersen SM, Chen S (2002) The relational self: an interpersonal social-cognitive theory. Psychol Rev 109:619-645.

Aron A, Aron EN, Tudor M, Nelson G (1991) Close relationships as including other in the self. J Pers Soc Psychol 60:241-253.

Barnett PA, Gotlib IH (1988) Psychosocial functioning and depression: distinguishing among antecedents, concomitants, and consequences. Psychol Bull 104:97-126.

Bartra O, McGuire JT, Kable JW (2013) The valuation system: a coordinatebased meta-analysis of BOLD fMRI experiments examining neural correlates of subjective value. Neuroimage 76:412-427.

Bates D, Mächler M, Bolker B, Walker S (2015) Fitting linear mixed-effects models using lme4. J Stat Softw 67:1-48.

Baumeister RF, Leary MR (1995) The need to belong: desire for interpersonal attachments as a fundamental human motivation. Psychol Bull 117:497529.

Baumeister RF, Tice DM (1990) Point-counterpoints: anxiety and social exclusion. J Soc Clin Psychol 9:165-195.

Benoit RG, Gilbert SJ, Volle E, Burgess PW (2010) When I think about me and simulate you: medial rostral prefrontal cortex and self-referential processes. Neuroimage 50:1340-1349.

Bergström ZM, Vogelsang DA, Benoit RG, Simons JS (2015) Reflections of oneself: neurocognitive evidence for dissociable forms of self-referential recollection. Cereb Cortex 25:2648-2657.

Berscheid E, Reis HT (1998) Interpersonal attraction and close relationships. In: Handbook of social psychology (Fiske S, Gilbert D, Lindzey G, Aronson E, eds), 4th ed, pp 193-281. New York: Random House.

Binder JF, Roberts SG, Sutcliffe AG (2012) Closeness, loneliness, support: core ties and significant ties in personal communities. Soc Netw 34:206214.

Branand B, Mashek D, Aron A (2019) Pair-bonding as inclusion of other in the self: a literature review. Front Psychol 10:2399.

Cacioppo JT, Hughes ME, Waite LJ, Hawkley LC, Thisted RA (2006) Loneliness as a specific risk factor for depressive symptoms: cross-sectional and longitudinal analyses. Psychol Aging 21:140-151.

Cacioppo JT, Norris CJ, Decety J, Monteleone G, Nusbaum H (2009) In the eye of the beholder: individual differences in perceived social isolation predict regional brain activation to social stimuli. J Cogn Neurosci 21:8392.

Chang LJ, Jolly E, Cheong JH, Rapuano K, Greenstein N, Chen PH, Manning JR (2018) Endogenous variation in ventromedial prefrontal cortex state dynamics during naturalistic viewing reflects affective experience. BioRxiv. doi: https://doi.org/10.1101/487892.
Chavez RS, Heatherton TF, Wagner DD (2017) Neural population decoding reveals the intrinsic positivity of the self. Cereb Cortex 27:5222-5229.

Chen PH, Wagner DD, Kelley WM, Powers KE, Heatherton TF (2013) Medial prefrontal cortex differentiates self from mother in Chinese: evidence from self-motivated immigrants. Cult Brain 1:3-15.

Clithero JA, Rangel A (2014) Informatic parcellation of the network involved in the computation of subjective value. Soc Cogn Affect Neurosci 9:1289-1302.

Cox RW, Chen G, Glen DR, Reynolds RC, Taylor PA (2017) fMRI clustering in AFNI: false-positive rates redux. Brain Connect 7:152-171.

Dale AM (1999) Optimal experimental design for event-related fMRI. Hum Brain Mapp 8:109-114.

Denny BT, Kober H, Wager TD, Ochsner KN (2012) A meta-analysis of functional neuroimaging studies of self- and other judgments reveals a spatial gradient for mentalizing in medial prefrontal cortex. J Cogn Neurosci 24:1742-1752.

Diener E, Seligman ME (2002) Very happy people. Psychol Sci 13:81-84.

Dunbar RI (2018) The anatomy of friendship. Trends Cogn Sci 22:32-51.

Eklund A, Nichols TE, Knutsson H (2016) Cluster failure: why fMRI inferences for spatial extent have inflated false-positive rates. Proc Natl Acad Sci USA 113:7900-7905.

Feng C, Yan X, Huang W, Han S, Ma Y (2018) Neural representations of the multidimensional self in the cortical midline structures. Neuroimage 183:291-299.

Galinsky AD, Ku G, Wang CS (2005) Perspective-taking and self-other overlap: fostering social bonds and facilitating social coordination. Group Process Intergroup Relations 8:109-124.

Granovetter MS (1973) The strength of weak ties. Am J Sociol 78:1360-1380.

Hanke M, Halchenko YO, Sederberg PB, Hanson SJ, Haxby JV, Pollmann S (2009) PyMVPA: a Python toolbox for multivariate pattern analysis of fMRI data. Neuroinformatics 7:37-53.

Hawkley LC, Cacioppo JT (2010) Loneliness matters: a theoretical and empirical review of consequences and mechanisms. Ann Behav Med 40:218-227.

Heatherton TF, Wyland CL, Macrae CN, Demos KE, Denny BT, Kelley WM (2006) Medial prefrontal activity differentiates self from close others. Soc Cogn Affect Neurosci 1:18-25.

Holt-Lunstad J, Smith TB, Layton JB (2010) Social relationships and mortality risk: a meta-analytic review. PLoS Med 7:e1000316.

Inagaki TK, Muscatell KA, Moieni M, Dutcher JM, Jevtic I, Irwin MR, Eisenberger NI (2016) Yearning for connection? Loneliness is associated with increased ventral striatum activity to close others. Soc Cogn Affect Neurosci 11:1096-1101.

Jones WH (1981) Loneliness and social contact. J Soc Psychol 113:295-296.

Jones WH, Hobbs SA, Hockenbury D (1982) Loneliness and social skill deficits. J Pers Soc Psychol 42:682-689.

Kang SK, Hirsh JB, Chasteen AL (2010) Your mistakes are mine: self-other overlap predicts neural response to observed errors. J Exp Soc Psychol 46:229-232.

Klinger E (1977) Meaning and void: inner experience and the incentives in people's lives. Minneapolis: University of Minnesota.

Kriegeskorte N, Mur M, Bandettini P (2008) Representational similarity analysis: connecting the branches of systems neuroscience. Front Syst Neurosci 2:4.

Krienen FM, Tu PC, Buckner RL (2010) Clan mentality: evidence that the medial prefrontal cortex responds to close others. J Neurosci 30:1390613915.

Kuznetsova A, Brockhoff PB, Christensen RH (2017) lmerTest package: tests in linear mixed-effects models. J Stat Softw 82:1-26.

Lieberman MD, Straccia MA, Meyer ML, Du M, Tan KM (2019) Social, self, (situational), and affective processes in medial prefrontal cortex (mPFC): causal, multivariate, and reverse inference evidence. Neurosci Biobehav Rev 99:311-328.

Meyer ML, Lieberman MD (2018) Why people are always thinking about themselves: medial prefrontal cortex activity during rest primes self-referential processing. J Cogn Neurosci 30:714-721.

Moran JM, Lee SM, Gabrieli JDE (2011) Dissociable neural systems supporting knowledge about human character and appearance in ourselves and others. J Cogn Neurosci 23:2222-2230.

Murray RJ, Schaer M, Debbané M (2012) Degrees of separation: a quantitative neuroimaging meta-analysis investigating self-specificity and shared 
neural activation between self- and other-reflection. Neurosci Biobehav Rev 36:1043-1059.

Parkinson C, Kleinbaum AM, Wheatley T (2018) Similar neural responses predict friendship. Nat Commun 9:332.

Parkinson CM, Kleinbaum AM, Wheatley T (2017) Spontaneous neural encoding of social network position. Nat Hum Behav 1:1-7.

Peirce JW (2008) Generating stimuli for neuroscience using PsychoPy. Front Neuroinform 2:10.

Poldrack RA, Mumford JA (2009) Independence in ROI analysis: where is the voodoo? Soc Cogn Affect Neurosci 4:208-213.

Roberts SG, Dunbar RI (2011) Communication in social networks: effects of kinship, network size, and emotional closeness. Personal Relationships 18:439-452.

Russell D, Peplau LA, Cutrona CE (1980) The revised UCLA Loneliness Scale: concurrent and discriminant validity evidence. J Pers Soc Psychol 39:472-480.

Russell DW, Cutrona CE, McRae C, Gomez M (2012) Is loneliness the same as being alone? J Psychol 146:7-22.

Sandstrom GM, Dunn EW (2014) Social interactions and well-being. Pers Soc Psychol Bull 40:910-922.

Schmitz TW, Johnson SC (2007) Relevance to self: a brief review and framework of neural systems underlying appraisal. Neurosci Biobehav Rev 31:585-596.

Seger CA, Stone M, Keenan JP (2004) Cortical activations during judgments about the self and other person. Neuropsychologia 42:1168-1177.

Segrin C, Flora J (2000) Poor social skills are a vulnerability factor in the development of psychosocial problems. Human Comm Res 26:489-514.

Spitzberg BH, Hurt HT (1987) The relationship of interpersonal competence and skills to reported loneliness across time. J Soc Behav Pers 2:157.

Stiller J, Dunbar RI (2007) Perspective-taking and memory capacity predict social network size. Soc Netw 29:93-104.

Takahashi H, Yahata N, Koeda M, Matsuda T, Asai K, Okubo Y (2004) Brain activation associated with evaluative processes of guilt and embarrassment: an fMRI study. Neuroimage 23:967-974.
Tamir DI, Mitchell JP (2012) Disclosing information about the self is intrinsically rewarding. Proc Natl Acad Sci USA 109:8038-8043.

Tavares RM, Mendelsohn A, Grossman Y, Williams CH, Shapiro M, Trope Y, Schiller D (2015) A map for social navigation in the human brain. Neuron 87:231-243.

Thornton MA, Mitchell JP (2017) Consistent neural activity patterns represent personally familiar people. J Cogn Neurosci 29:1583-1594.

Thornton MA, Weaverdyck ME, Mildner JN, Tamir DI (2019) People represent their own mental states more distinctly than those of others. Nat Commun 10:2117.

Van der Laan M, Pollard K, Bryan J (2003) A new partitioning around medoids algorithm. J Stat Comput Simul 73:575-584

Vul E, Harris C, Winkielman P, Pashler H (2009) Puzzlingly high correlations in fMRI studies of emotion, personality, and social cognition. Perspect Psychol Sci 4:274-290.

Wagner DD, Chavez RS, Broom TW (2019) Decoding the neural representation of self and person knowledge with multivariate pattern analysis and data-driven approaches. Wiley Interdiscip Rev Cogn Sci 10:e1482.

Wagner DD, Haxby JV, Heatherton TF (2012) The representation of self and person knowledge in the medial prefrontal cortex. Wiley Interdiscip Rev Cogn Sci 3:451-470.

Wellman B, Wortley S (1990) Different strokes from different folks: community ties and social support. Am J Sociol 96:558-588.

Wittenberg MT, Reis HT (1986) Loneliness, social skills, and social perception. Pers Soc Psychol Bull 12:121-130.

Yamada M, Decety J (2009) Unconscious affective processing and empathy: an investigation of subliminal priming on the detection of painful facial expressions. Pain 143:71-75.

Yarkoni T, Poldrack RA, Nichols TE, Van Essen DC, Wager TD (2011) Large-scale automated synthesis of human functional neuroimaging data. Nat Methods 8:665-670.

Zhou WX, Sornette D, Hill RA, Dunbar RI (2005) Discrete hierarchical organization of social group sizes. Proc Biol Sci 272:439-444. 